\title{
4
}

\section{Modeling Defibrillation}

\author{
Gernot Plank ${ }^{1}$ and Natalia Trayanova ${ }^{2}$ \\ ${ }^{1}$ Medical University of Graz, Austria \\ ${ }^{2} J o h n s$ Hopkins University, MD, Baltimore \\ ${ }^{1}$ Austria \\ ${ }^{2} U S A$
}

\section{Introduction}

Cardiac fibrillation is the breakdown of the organized electrical activity in the heart into disorganized self-sustained electrical activation patterns. Fibrillatory episodes when affecting the ventricles, i.e. the main pumping chambers of the heart, result in loss of cardiac output and unless timely intervention is administered, death quickly ensues. The only known effective therapy for lethal disturbances in cardiac rhythm is defibrillation, the delivery of a strong electric shock to the heart. This technique, as accomplished nowadays by automatic, implantable cardioverter-defibrillator (ICD) devices, constitutes the most important means of combating sudden cardiac death. Several multi-center clinical trials have provided consistent evidence that ICD therapy prolongs patient life. This convincing demonstration of efficacy has led to a nearly exponential growth, over the last decade, in the number of patients receiving implantable devices. Currently, around 0.2 million ICDs are implanted every year throughout the world.

Although ICD therapy has proved to be efficient and reliable in preventing sudden cardiac death (Bardy et al., 1993), with success rates clearly superior to other therapeutic options such as pharmacological anti-arrhythmia therapy (Zipes et al., 1997), it is far from ideal. There are several known adverse effects secondary to the administration of electrical shocks, the most prominent are linked to electroporation (DeBruin \& Krassowska, 1998), (i.e. the formation of pores in the cellular membrane that allow the free and indiscriminate redistribution of ions, enzymes and large molecules between intracellular and interstitial space), and its after-effects which are indirectly caused by the high field strengths required to terminate arrhythmias such as ventricular fibrillation (VF) with sufficiently high probability. Moreover, psychological effects on patients play an important role. Conscious patients may perceive shock delivery as extremely painful which leads to traumatization and reduction in quality of life. Although pain may be tolerable in those cases where shock delivery saves human lives, this is less likely in those cases where inadequate shocks are delivered due to high voltage component malfunctions of the device. A recent meta-analysis of industrial reports (Maisel, 2006) concluded that such malfunctions are much more frequent than expected, with thousands of patients being affected. Further, clinical data from ICD trials suggested that 6 out of 7 shocks delivered can be classified as inadequate, indicating the amount of over-treatment in the ICD population is significant (Zipes et al., 1997). Therefore, despite the impressive clinical success and efficacy of ICD implant, the therapy is clearly suboptimal due to these numerous adverse side effects and as such research on defibrillation mechanisms remains 
to be an important topic. Achieving defibrillation reliably with shocks of much lower energy than those delivered by ICD devices today, would constitute a major step forward. Advancing our understanding towards a full appreciation of the mechanisms by which a shock interacts with the heart is the most promising approach to achieve this goal.

\subsection{Experimental approaches to investigate defibrillation mechanisms}

Elucidating the biophysical underpinnings of defibrillation mechanisms has been a long and arduous process. The key to understanding the cardiac defibrillation process is to uncover those mechanisms by which electric current delivered to the heart during shock delivery traverses myocardial structures and interacts with the wavefronts of fibrillation. The quest for uncovering these mechanistic links between applied electric fields and evoked membrane responses has proved to be enormously challenging. In no small part these difficulties can be attributed to the lack of suitable experimental methodologies which would allow to observe electrical events occurring before, during and after shock delivery with sufficiently high spatio-temporal resolution. Early studies relied on recordings of extracellular potentials following the defibrillation shock, since overwhelming electrical artifacts had prevented researchers from recording during and shortly after the shock. Although these pioneering electrical mapping studies provided some insights which laid the basis for developing the first theories such as the upper limit of vulnerability (ULV) hypothesis, there was no direct experimental evidence to prove the putative mechanisms. A major breakthrough occur ed with the introduction of potentiometric dyes, which allowed to record electrical events before, during and after shock delivery at a higher spatio-temporal resolution. The ability of these optical mapping techniques to provide optical fluorescence signals, $V_{o p t}$, which are proportional to the transmembrane voltage, $\propto V_{m}$, proved to be a further advantage. Unlike extracellularly recorded electric potentials, $\Phi_{e}$, which integrate contributions of bioelectric activity in the vicinity of a recording site via the volume conductor in which the heart is immersed, $V_{m}$ provides a more direct measure for shock-induced membrane responses, although optical signals are not purely local representations of $V_{m}$ at a recording site either, since fluorescence detectors collect photons emerging from within a certain scattering volume (Bishop et al., 2007). Although optical mapping setups allow to elegantly visualize shock-induced changes in tissue polarizations, these observations are confined to record electrical activity from cardiac surfaces only. However, in a complex three-dimensional anatomical structure such as the heart electrical events occur throughout the entire myocardium including the depth of the myocardial walls. Therefore, the restricted capabilities of current optical mapping techniques to detect events which may occur in deeper layers of the myocardial wall without any signature at the surfaces (Ashihara et al., 2008), poses a severe limitation, rendering investigations of defibrillation mechanisms by experimental means alone a challenging endeavor.

\subsection{Role of computer modeling}

The absence of experimental methodology for observing shock-induced membrane polarizations in 3D inspired the theoretical community to develop and refine computer models of the cardiac defibrillation process. Since there is insufficient direct experimental evidence which would allow a direct verification of model predictions in the depth of the myocardium, computer models are compared against optical maps recorded at the ventricular surfaces. Assuming that model formulations are valid quantitatively correct representation of cardiac bioelectricity, models which correctly predict experimental observations at the 


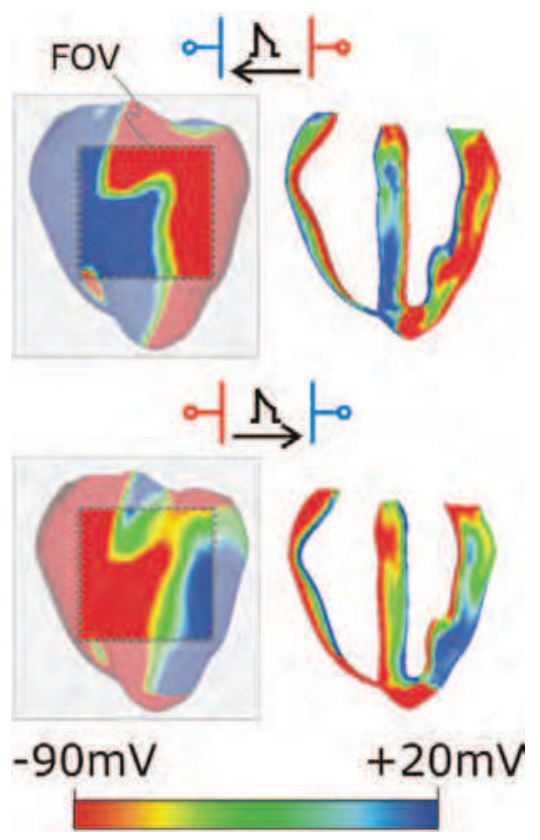

Fig. 1. Left panel: In most experimental setups the field of view (FOV) of optical recordings, as indicated by a gray dashed rectangle, is confined to the surface. Shown are polarization patterns at the end of the shock delivered via plate electrodes, with the cathode being located next to the left ventricle (top) and the right ventricle (bottom). Right panel: Computer models allow to observe shock-induced changes in polarization throughout the entire myocardium, revealing important shock-induced changes in electrical state of the tissue, such as shape, size and location of the post-shock excitable gap (blue regions).

surfaces could be used with some confidence to take a look inside the depth of myocardial walls, thus allowing to bridge the gap between experimental observations recorded at epicardial and endocardial surfaces (Fig. 1).

In earlier computational studies of defibrillation mechanisms monodomain models were employed. Such monodomain formulations do not account explicitly for current flow in the extracellular domains. This simplification reduces model complexity and computational costs at the cost of biophysical accuracy. Although monodomain models were well established for studying impulse propagation in the heart, their suitability in the context of simulating the process of defibrillation quickly turned out to be of limited value. Theoretical considerations based on the monodomain equations predicted shock-induced changes in transmembrane voltage, $\Delta V_{m}$, only along tissue boundaries and around conductive discontinuities in the heart, leaving the bulk of the myocardium essentially unaffected. However, such predictions contradicted experimental findings which had established that a critical mass of the tissue of $\sim 95 \%$ (Adgey et al., 2005; Ideker et al., 1991) has to be affected by a sufficiently strong gradient of $>5 \mathrm{~V} / \mathrm{cm}$ (Frazier et al., 1989; Zhou et al., 1993) to be effective. To resolve this dilemma the theoretical community put forward the more comprehensive bidomain model which explicitly accounts for current flow in both the intracellular and interstitial domains. The bidomain model proved quickly to be an invaluable powerful tool for studying defibrillation 
mechanisms, providing the sought after "missing link" (Roth \& Krassowska, 1998) between externally applied electric field and membrane responses in the tissue bulk. Using a bidomain model Sepulveda and coworkers (Sepulveda et al., 1989) demonstrated in a seminal study that shock-induced changes in membrane polarizations can be much more complex than previously anticipated. Their simulation results suggested that the tissue response in the vicinity of a strong unipolar stimulus involved the simultaneous occurrence of both positive (depolarizing) and negative (hyperpolarizing) effects in close proximity, if the anisotropy ratios between intracellular and interstitial space comprising the myocardium are unequal, i.e. both spaces are anisotropic, but to a different degree. In the absence of unequal anisotropy ratios, as it is the case with monodomain models which inherently assume equal anisotropies, no polarizations of opposite polarity can occur (Fig. 2). This prediction of the existence of
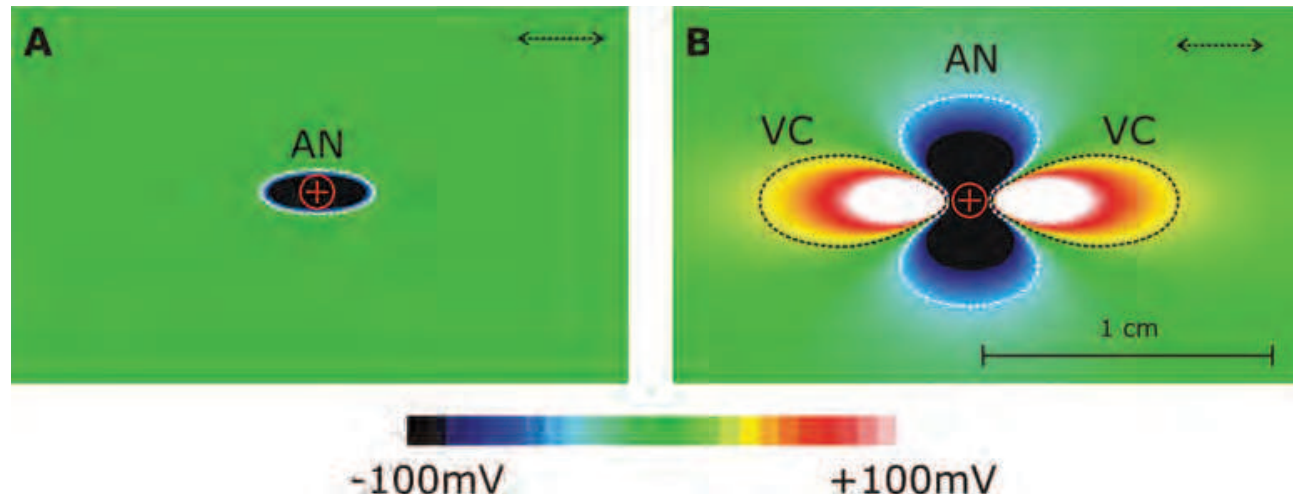

Fig. 2. Unequal anisotropy ratios between intracellular and interstitial space is a necessary condition for the existence of bulk VEPs. A) A unipolar anodal stimulus is delivered to the center of a tissue sheet of equal anisotropy ratios. Shock induced polarizations of only one polarity (hyperpolarizing) are observed and the tissue affected by the shock is limited the immediate vicinity of the electrode. B) The exact same shock delivered to tissue of unequal anisotropy ratios induced polarizations of both polarities and the area of tissue affected extends far beyond the electrotonic space constant. Dashed lines indicate the longitudinal axis of myocyte orientation.

"virtual electrodes" departed from the established view that tissue responses should only be depolarizing if the stimulus is cathodal, or hyperpolarizing if the stimulus is anodal. Optical mapping studies that followed convincingly confirmed these theoretical predictions (Wikswo Jr et al., 1995). Since, "virtual electrode polarization" (VEP) has been documented in experiments involving various stimulus configurations (Efimov, Aguel, Cheng, Wollenzier \& Trayanova, 2000; Efimov et al., 1997; Efimov, Gray \& Roth, 2000; Evans et al., 2002; Knisley et al., 1999).

\subsection{Current understanding of defibrillation mechanisms}

Conceptually, defibrillation can be considered to be a two-step process. First, the applied shock drives currents that traverse the myocardium and cause complex polarization changes in transmembrane potential distribution (Sobie et al., 1997). Secondly, post-shock active membrane reactions are invoked that eventually result either in termination of fibrillation in the case of shock success, or in reinitiation of fibrillatory activity in the case of shock 
failure. Using computer models to analyze the etiology of VEP patterns during the shock application phase revealed that shape, location, polarity, and intensity of shock-induced VEP are determined by both the cardiac tissue structure as well as the configuration of the applied field (Knisley et al., 1999; Rodriguez et al., 2005; Sobie et al., 1997). Based on theoretical considerations VEPs can be classified either as "surface VEP", which penetrates the ventricular wall over a few cell layers, or as "bulk VEP" where polarizations arise throughout the ventricular wall (Entcheva et al., 1999; Trayanova et al., 1998). Analysis of the bidomain equations revealed that a necessary condition for the existence of the bulk VEP is the presence of unequal anisotropies in the myocardium. Sufficient conditions include either spatial non-uniformity in applied electric field, or non-uniformity in tissue architecture, such as fiber curvature, fiber rotation, fiber branching and anastomosis, and local changes in tissue conductivity due to resistive heterogeneities (Plank et al., 2005) (Fig. 3).

The cellular response depends on VEP magnitude and polarity as well as on pre-shock state of the tissue. APD can be either extended (by positive VEP) or shortened (by negative VEP) to a degree that depends on VEP magnitude and shock timing, with strong negative VEP completely abolishing (de-exciting) the action potential thus creating post-shock excitable gaps. As demonstrated in bidomain modeling studies (Ashihara et al., 2008; Roth, 1995), the post-shock VEP pattern is also the major determinant of the origin of post-shock activations. In those regions where shock-induced virtual anodes and virtual cathodes are in close proximity, a "break" excitation at shock-end (i.e, the "break" of the shock) can be elicited. The virtual cathode serves as an electrical stimulus eliciting a regenerative depolarization and a propagating wave in the newly created excitable area. Whether or not break excitations arise depends on whether the transmembrane potential gradient across the border spans the threshold for regenerative depolarization (Cheng, Mowrey, Van Wagoner, Tchou \& Efimov, 1999). The finding of break excitations, combined with the fact that positive VEP can result in "make" excitations (where "make" refers to the onset of a shock) in regions where tissue is at or near diastole, resulted in a novel understanding of how a strong stimulus can trigger the development of new activations.

According to VEP theory, mechanisms for shock success or failure are multifactorial depending mainly on post-shock distribution of $V_{m}$ as well as timing and speed of propagation of shock-induced wavefronts. Whether the depolarization of the post-shock excitable gap is achieved in time critically depends on number and conduction velocity of post-shock activations, as well as the available time window which is bounded by the instant at which refractory borders enclosing the excitable regions recover excitability. All factors depend, ultimately, on shock strength. Increasing shock strength results in higher voltage gradients across borders between regions of opposite polarity, leading to more break-excitations (Cheng, Mowrey, Van Wagoner, Tchou \& Efimov, 1999) which then start to traverse the post-shock excitable gap earlier (Skouibine et al., 2000a) and at a faster velocity (Cheng, Mowrey, Van Wagoner, Tchou \& Efimov, 1999), as well as extending refractoriness to a larger degree (Knisley et al., 1994).

\section{Computationally modeling defibrillation and shock-induced arrhythmogenesis}

Comprehensive mechanistic insight into defibrillation remains a major scientific frontier and the 3D bidomain model is an important tool to complement and interpret experimental observations. The quest to unravel how shocks could succeed in terminating fibrillation or how they could re-instate arrhythmia has driven the technological aspects of computer simulations of $3 \mathrm{D}$ bidomain activity. In order to be able to simulate electrical processes 


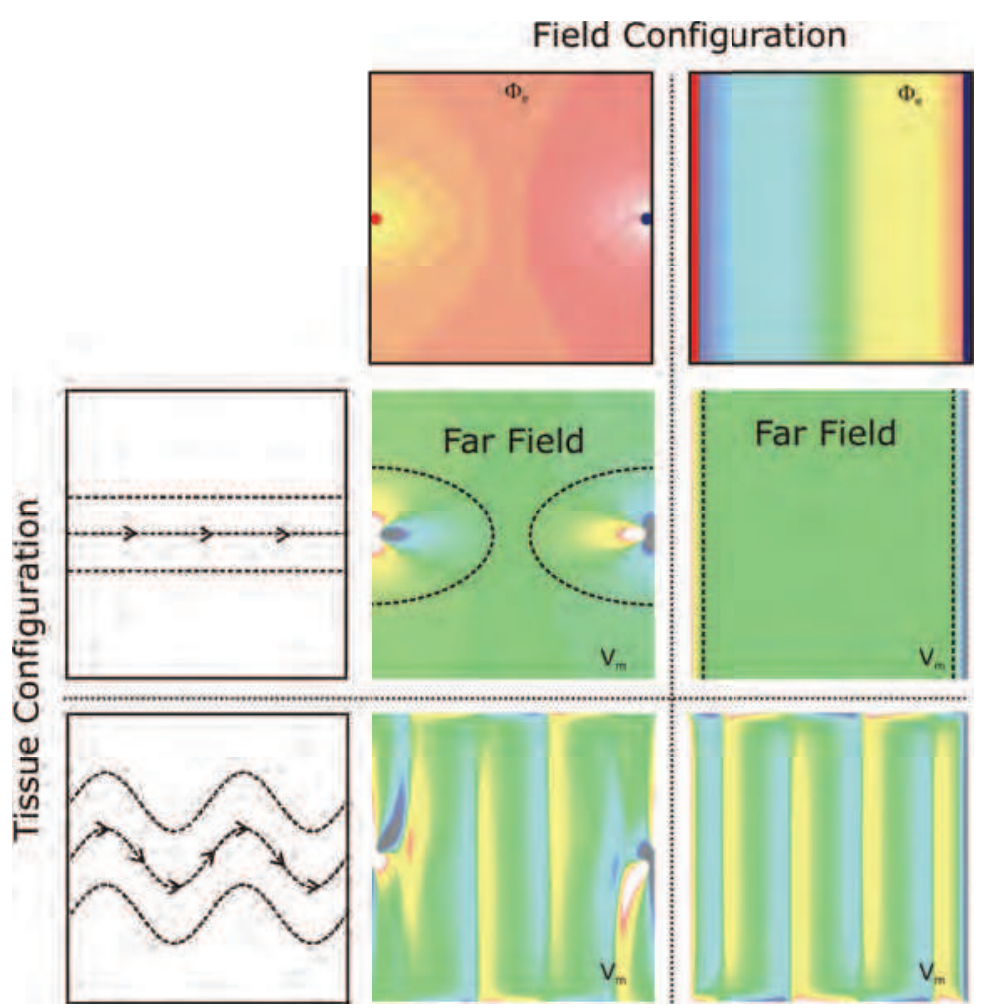

Fig. 3. An important insight learned from the bidomain model is that the etiology of VEPs is determined by both field configuration as well as tissue structure. Shown are shock-induced polarization patterns as a function of field and tissue configuration. Top panels: Shown are the extracellular electric potentials $\Phi_{e}$ as induced by two point-like electrodes (left) and line-like electrodes (right). Red(blue) indicates cathodal (anodal) stimulus. Left panels: Two tissue configurations are shown, a homogeneous configuration with straight fibers only (top), and a configuration where fiber orientation varies as a function of space (bottom). Preferred longitudinal axes of the tissue is indicated by dashed lines. Central panels: Shock-induced polarization patterns for all possible combinations between field and tissue configuration. In the case of plate electrodes with a homogeneous tissue structure only surface polarizations close to the electrode locations are observed. The bulk of the tissue remains essentially unaffected.

driven by the delivery of shocks to the ventricles, computational research has managed to overcome tremendous difficulties associated with obtaining solutions of very large systems of unknowns, involving stiff equations and computational meshes of irregular geometry. A brief overview of the computational approaches involved in conducting simulations of shock administration and post-shock arrhythmogenesis is presented below.

\subsection{Simulating cardiac bioelectric activity at the tissue and organ level}

Computer models of cardiac biolelectric activity are built upon first principles which represent the movement of ions in within the myocardial tissue. A building block of central importance 
are cardiac myocytes which make up the tissue. A myocyte is of roughly cylindrical shape, $\sim 100 \mu \mathrm{m}$ long and $10-20 \mu \mathrm{m}$ in diameter. The intracellular spaces of adjacent myocytes are interconnected by specialized connexins referred to as gap junctions (Desplantez et al., 2007). The connexin expression over the cell is heterogeneous with a higher density of gap junctions at the intercalated discs located at the cell ends (along the long axis of the cell) and a lower density along the lateral boundaries (Gourdie et al., 1991; Hoyt et al., 1989), and different connexins of varying conductance are expressed in different regions of the heart (Severs et al., 2008). As a consequence of the elongated cellular geometry as well as the directionally varying gap junction density current flows more readily along the longitudinal axes of the cells than transverse to it. This property is referred to as anisotropy. Cardiac tissue is composed of two spaces: an intracellular space formed by the interconnected network of myocytes, and the cleft spaces between myocytes referred to as interstitial space which is made up of the extracellular matrix and the interstitial fluid. The extracellular matrix consists of networks of collagen fibers which determine the passive mechanical properties of the myocardium. Electrically, it is assumed that the preferred directions are co-aligned between the two spaces, but that the conductivity ratios between the principal axes are unequal between the two domains (Clerc, 1976; Hooks et al., 2007; Roberts \& Scher, 1982; Roth, 1997). All parameters influencing the electrical properties of the tissue, such as density and conductance of gap junctions, cellular geometry, orientation and cell packing density, and the composition of the interstitial space, are heterogeneous and may vary at all size scales, from the cellular level up to the organ. As a consequence, direction and speed of a propagating electric wave is constantly modified by interactions with discontinuous spatial variations in material properties at various size scales. At a finer size scale below the space constant, $\lambda$, of the tissue, i.e. $<1 \mathrm{~mm}$, the tissue is best characterized as a discrete network in which the electrical impulse propagates in a discontinuous manner (Kleber \& Rudy, 2004; Spach \& Heidlage, 1995). At a larger more macroscopic size scale $(\gg \lambda)$ the tissue behaves as a continuous functional syncytium where the effects of small-scale discontinuities are assumed to play a minor role.

Theoretically, the idea of modeling an entire heart by using models of a single cell as the basic building block is conceivable. However, the associated computational costs are prohibitive with current computing hardware, since a single heart consists of roughly 5 billion cells. Although a few high resolution modeling studies have been conducted where small tissue preparations were discretized at a sub-cellular resolution (Hooks et al., 2007; Roberts et al., 2008; Spach \& Heidlage, 1995), in general the spatial discretization steps were chosen based on the spatial extent of electrical wave fronts and not on the size scales of the tissue's micro-structures. For this sake cardiac tissue is treated as a continuum for which appropriate material parameters have to be determined which translate the discrete cellular matrix into an electrically analog macroscopic representation. In principle, this is achieved by averaging material properties over suitable length scales such that both potential and current solution match between homogenized and discrete representation. A rigorous mathematical framework for this procedure is provided by homogenization theory which has been applied by several authors to the bidomain problem (Hand et al., 2009; Henriquez, 1993; Neu \& Krassowska, 1993; Pennacchio M. et al., 2006). Homogenization is a two-step process where the intracellular and interstitial domain are homogenized in a first step and the two respective domains are spread out and overlapped to fill the entire tissue domain. This concept of interpenetrating domains states that everywhere within the entire myocardial volume intracellular space, extracellular space and the cellular membrane coexist (Fig. 4). 


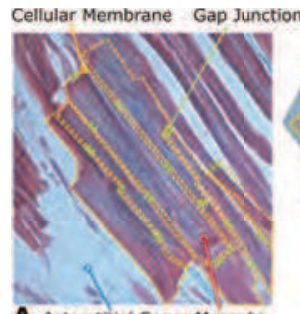

A Interstitial Space Myocyte

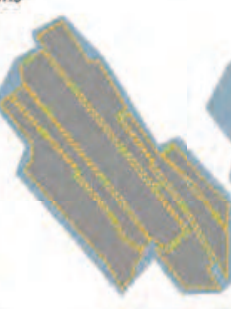

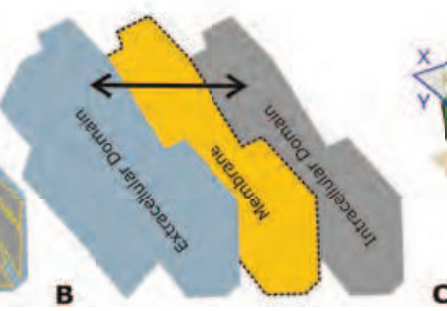

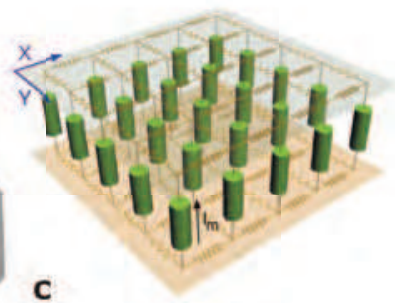

Fig. 4. a) Cardiac tissue is made up of discrete myocytes which are interconnected via gap junctions. b) Discrete structures in both the intracellular as well as the interstitial space are homogenized to arrive at a continuum representation, which matches electrical properties between discrete microscopic size scale and continuous macroscopic size scale. Further, both homogenized domains are overlapped, separated by a membrane at each point in space (interpenetrating domains). c) Discrete representation of both conductive domains and membrane, as discretized by a finite difference approach.

\subsection{Governing equations}

The bidomain equations(Plonsey, 1988) describe the electrical behavior of cardiac tissue as a syncytium, where all tissue parameters are accounted for in an averaged sense. The domains of interest, intracellular and extracellular, and the cellular membranes which physically separate the two domains, are distributed over the entire tissue volume. The bidomain equations state that currents that enter the intracellular or extracellular spaces by crossing the cell membrane represent the sources for the intracellular potential, $\phi_{i}$, and the extracellular potential, $\phi_{e}$,

$$
\begin{aligned}
\nabla \cdot \boldsymbol{\sigma}_{\boldsymbol{i}} \nabla \phi_{i} & =\beta I_{m} \\
\nabla \cdot \boldsymbol{\sigma}_{\boldsymbol{e}} \nabla \phi_{e} & =-\beta I_{m}-I_{e} \\
I_{m} & =C_{m} \frac{\partial V_{m}}{\partial t}+I_{i o n}\left(V_{m}, \boldsymbol{\eta}\right)-I_{\text {stim }} \\
V_{m} & =\phi_{i}-\phi_{e},
\end{aligned}
$$

where $\sigma_{i}$ and $\sigma_{e}$ are the intracellular and extracellular conductivity tensors, respectively, $\beta$ is the bidomain membrane surface to volume ratio, $I_{m}$ is the transmembrane current density, $I_{\text {stim }}$ is the current density of the transmembrane stimulus used to initiate an action potential, $I_{e}$ is the current density of the extracellular stimulus, $C_{m}$ is the membrane capacitance per unit area, $V_{m}$ is the transmembrane potential, and $I_{i o n}$ is the density of the total current flowing through the membrane ionic channels, pumps and exchangers, which depends on $V_{m}$ and a set of state variables, $\boldsymbol{\eta}$. At the tissue boundaries, electrical isolation is assumed, which is accounted for by imposing no-flux boundary conditions on $\phi_{e}$ and $\phi_{i}$.

If, however, cardiac tissue is surrounded by a conductive medium, such as blood in the ventricular cavities or a perfusing bath (Tyrode solution) in which the heart is submerged, then Laplace equation has to be additionally solved

$$
\nabla \cdot \sigma_{b} \nabla \phi_{e}=0
$$


where $\sigma_{b}$ is the isotropic conductivity of the conductive medium. In this case no-flux boundary conditions are assumed at the boundaries of the conductive medium, whereas continuity of the normal component of the extracellular current and continuity of $\phi_{e}$ are enforced at the tissue-bath interface. The no-flux boundary conditions for $\phi_{i}$ remain the same.

For most applications the bidomain equations are recast into other forms by substituting eq.(4) into (1) and (2) and executing algebraic transformations. Several ways to recast the bidomain equations have been proposed; a systematic overview of the different linear transformations is found in (Hooke et al., 1994). A widely used transformation is to add eqs. (1) and (2) and replace $\phi_{i}$ by $V_{m}+\phi_{e}$ (Pollard et al., 1992)

$$
\begin{aligned}
\nabla \cdot\left(\boldsymbol{\sigma}_{\boldsymbol{i}}+\boldsymbol{\sigma}_{\boldsymbol{e}}\right) \nabla \phi_{e} & =-\nabla \cdot \boldsymbol{\sigma}_{\boldsymbol{i}} \nabla V_{m}-I_{e} \\
\nabla \cdot \boldsymbol{\sigma}_{\boldsymbol{i}} \nabla V_{m} & =-\nabla \cdot \boldsymbol{\sigma}_{\boldsymbol{i}} \nabla \phi_{e}+\beta I_{m},
\end{aligned}
$$

which retains $V_{m}$ and $\phi_{e}$ as the independent variables. For comparison of tissue and organ level simulations with experimental data this is advantageous since $\phi_{e}$ can be measured via electrical mapping techniques and optical mapping techniques allow to record signals $V_{\text {opt }} \propto$ $V_{m}$.

\subsection{Computational considerations}

Large-scale computational studies employing the bidomain model in general, and defibrillation studies in particular, have remained a challenge even though computer speed and memory have dramatically increased. There are numerous factors which render any numerical solution of the bidomain equations computationally challenging. First, the upstroke of the action potential is very fast, lasting only $\sim 1 \mathrm{~ms}$. These fast transients translate into a steep propagating wavefront in space where the depolarization wavefront extends only a few hundreds of $\mu \mathrm{m}$. As a consequence, both spatially fine-grained computational grids and a high temporal resolution is required to faithfully capture wavefront propagation. Further, the discretized domain of interest has to be chosen large enough to support reentrant wave propagation when studying the formation, maintenance and termination of cardiac arrhythmias. With constraints on spatial discretization as mentioned above, the results is a large system, on the order of 0.1 to 100 million degrees of freedom. Finally, the maximum time step which can be taken to advance the solution of the bidomain equations in time is limited, either by stability (Courant et al., 1928) or accuracy constraints. Since the physiological processes of interest take place over seconds or minutes, temporal step size limits necessitate a large number of time steps, typically in the range from tens to hundreds of thousands.

Beyond the high computational load imposed by bidomain simulations in general, there are numerous additional methodological challenges which need to be addressed when using computer models for studying the delivery of defibrillation shocks. To model the effect of extracellularly applied fields, the use of unstructured grids for anatomically realistic models of the heart is mandatory as to allow smooth representation of the organ's surfaces. Jagged boundaries, which inevitably form along the organ surfaces when regular structured or block structured grids are employed, cause spurious polarizations upon delivery of a defibrillation-strength shock. Further, even finer spatial discretization may be required since large transmembrane voltage gradients are induced by the shock; for instance, in a passive 2D bidomain study (Aguel et al., 1999) a voltage drop of $1 \mathrm{~V}$ over a distance as short as 100 $\mu \mathrm{m}$ has been reported. Another serious difficulty is the use of state-of-the-art ionic models which incorporate now up to several tens of state variables of ever increasing stiffness. These models are developed and tested within the normal physiological range of action potentials, 
however, during the shock administration transmembrane voltages may rise significantly beyond this range, even when ionic models are augmented with additional currents such as electroporation currents (DeBruin \& Krassowska, 1998) or hypothetical potassium currents (Cheng, Tung \& Sobie, 1999). Although these currents kick in at elevated transmembrane voltages to cap the rise of $V_{m}$ at a few hundreds of $\mathrm{mV}$, transmembrane voltages still rises well beyond the physiological range which could potentially entail undesirable behavior of the model equations. Moreover, due to the even faster transients in state variables during shock onset enforce, even smaller time steps may be required, making computations during the shock very burdensome. Typically, modifications are required to render an ionic model suitable for defibrillation studies (Ashihara \& Trayanova, 2004; Skouibine et al., 2000b). Finally, due to the nature of defibrillation, where shock success depends on a multitude of parameters such as shock strength and timing, pulse shape and polarity or electrode geometry and location, a large number of simulations is required to sweep the parameter space. For instance, a standard problem is to determine the window of vulnerability for a given electrode configuration, that is, for which range of shock strength and coupling intervals the tissue is vulnerable to arrhythmia induction. Such studies involve the construction of vulnerability grids where $N$ timings and $M$ shock strengths have to be tested. Therefore a total of $N \times M$ shocks need to be computed and $N \times M$ simulations of post-shock evolution need to be performed to determine arrhythmia inducibility for each combination.

\subsection{Spatial discretization}

Various spatial discretization techniques have been applied to the cardiac bidomain problem, most notably the finite difference method (FDM) (Potse et al., 2006; Skouibine et al., 2000a), the finite volume method (FVM) (Harrild \& Henriquez, 1997; Trew, Le Grice, Smaill \& Pullan, 2005) and the finite element method (FEM) (Rogers \& McCulloch, 1994; Vigmond et al., 2002), although other non-standard techniques such as the interconnected cable model have been employed successfully as well (Leon \& Roberge, 1991; Wang et al., 1996). In general, the FDM is easiest to implement, but the method does not accommodate complex boundaries as naturally as the FEM or the FVM do. Although suggestions were made to overcome this limitation by employing the phase-field approach (Fenton et al., 2005) or other generalizations (Buist et al., 2003; Trew, Smaill, Bullivant, Hunter \& Pullan, 2005), the FDM looses its most appealing advantage, the ease of implementation. FEM and FVM are both very well suited for spatial discretizations of complex geometries with smooth representations of the boundaries, which is a key feature when polarization patterns induced via extracellularly applied currents are to be studied. Both FVM and FEM have been used to model electrical activity in anatomical realistic models of the atria (Harrild \& Henriquez, 2000; Seemann et al., 2006; Vigmond et al., 2004; Virag et al., 2002) as well as the ventricles (Ashihara et al., 2008; Plank et al., 2009; Potse et al., 2006; Ten Tusscher et al., 2007). Mesh generation requirements are similar for both techniques, that is, the domain of interest has to be tessellated into a set of non-overlapping and conformal geometric primitives (Fig. 5). With the FVM, quadrilaterals in 2D (Harrild \& Henriquez, 1997) and hexahedral elements in 3D (Harrild \& Henriquez, 2000; Trew, Le Grice, Smaill \& Pullan, 2005) have been preferred, whereas with the FEM, triangles and quadrilaterals were used in 2D and tetrahedral (Plank et al., 2009) or hexahedral elements in 3D (Munteanu et al., 2009; Seemann et al., 2006). Typically, monolithic meshes consisting of one element type only were used, but exception exist (Prassl et al., 2009; Rocha et al., 2011) where hybrid meshes consisting of tetrahedra, hexahedra, pyramids and prisms were used. Further, most FEM studies relied on the Galerkin FEM where linear test functions 
with tetrahedral elements (Franzone et al., 2006; Sundnes et al., 2006; Vigmond et al., 2002), isoparametric trilinear test functions with hexahedral elements (Munteanu et al., 2009) or cubic-hermite hexahedral elements (Rogers \& McCulloch, 1994; Saucerman et al., 2004) were used.

Independently of the spatial discretization technique, the choice of space step, $h$, is of major importance. It is known since very early modeling studies that the solution of the bidomain equations does depend, to a certain degree, on $h$, even with very fine spatial discretizations (Pollard et al., 1993). This sensitivity has to be attributed to the non-linearity and stiffness of the reaction term which entails an extremely fast upstroke of the cardiac action potential, lasting $\sim 1 \mathrm{~ms}$ only. When propagating, a fast upstroke in time translates into a steep wavefront in space. Depending on tissue conductivity and cellular excitability, physiological conduction velocities range between $0.2-0.7 \mathrm{~m} / \mathrm{s}$ within the myocardium which translates an upstroke duration of $1 \mathrm{~ms}$ duration into a wavefront that extends $200-700 \mu \mathrm{m}$ in space. Under pathological situations where tissue conductivity and/or excitability is reduced, conduction velocity may be substantially slower, leading to wavefronts where the spatial extent may be even below $100 \mu \mathrm{m}$. The spatial extent of a wavefront along a direction $\zeta$ is proportional to the space constant, $\lambda_{\zeta}$

$$
\lambda_{\zeta}=\sqrt{\frac{1}{\beta} \frac{\sigma_{i \zeta} \sigma_{e \zeta}}{\sigma_{i \zeta}+\sigma_{e \zeta}}} .
$$

It has been shown that for sufficiently small effective discretizations, $H_{\zeta}=\lambda_{\zeta} / h_{\zeta}<0.15$, solutions converge with deviations in conduction velocity $<1 \%$ (Pollard et al., 1993). In practice, a trade-off has to be made between accuracy and computational tractability. In tissue and organ scale modeling studies a standard choice for $h$, or for an average discretization $\bar{h}$ when unstructured grids are considered, is $250 \mu \mathrm{m}$, but finer (Plank et al., 2009) as well as coarser discretizations (Ashihara et al., 2008; Saucerman et al., 2004) have been reported as well. With very coarse discretizations, $h>500 \mu \mathrm{m}$, and physiologically realistic models of cellular dynamics simulations deviate substantially from results obtained at finer resolutions. Conduction velocities at such coarse grids are underestimated to different degrees as a function of direction, leading to wavefront distortions (Clayton et al., 2011), even conduction block may occur as a numerical side effect due to spatial undersampling.

\subsection{Construction of models of cardiac anatomy}

In order to construct geometrically-realistic models of cardiac anatomy, such information must be first obtained via various different imaging modalities, to then be processed and used in model construction. In the past decade or so, efforts have been focused towards developing techniques to construct 3D computational cardiac models directly from non-invasive 3D imaging modalities such as magnetic resonance (MR). In the last few years, the advent of stronger magnets and refined scanning protocols has significantly increased the resolution of anatomical MR scans, such that small mammalian hearts now can have MR voxel dimensions of $\approx 20-25 \mu \mathrm{m}$ (Burton et al., 2006; Plank et al., 2009). An example of a high resolution anatomical MR scan of a rabbit heart with voxel resolution $\approx 25 \mu \mathrm{m}$ isotropic is shown in Fig. 5. As a result of this increase in attainable resolution, anatomical MR imaging is now capable of providing a wealth of information regarding fine-scaled cardiac structural complexity. Such MR data is currently allowing accurate identification of microscopic features such as the coronary vasculature, extracellular cleft spaces and the free-running Purkinje system, as well as macroscopic structures such as trabeculations and papillary muscles. In addition, information regarding the organization of cardiomyocytes into cardiac fibers 
(Streeter et al., 1969), as well as the laminar structure of the myocardial wall (LeGrice et al., 1995) is required to account for orthotropic tissue properties. Such data is unattainable with normal anatomical MR imaging, however, the eigenaxes of the tissue can be estimated in 3D using diffusion-tensor MR imaging (DT-MRI).

This information must then be processed and transformed into a usable format to facilitate the generation of anatomically-detailed computational cardiac models. A first processing step is to faithfully extract the complex geometrical information present in the image stacks. This procedure, referred to as segmentation, involves labeling voxels based-on their association with different regions, types of tissue, objects or boundaries within the image. Ideally, computational algorithms are employed, which automatically segment regions of interest within the image with little or no manual input. For generating a computational model it is required to discriminate those voxels in the MR data set which belong to cardiac 'tissue' from those which represent non-tissue or 'background', effectively translating a gray-scale MR image data set into a binary black/white $(0 / 1)$ image mask.

In a final step, classified objects in the binarized image stacks are tessellated into finite element meshes. The construction of such meshes is a highly non-trivial task. Recent advances in image-based mesh generation techniques allow the direct construction of finite element meshes using segmented image stacks as input (Prassl et al., 2009). Although the exceptionally high resolution of such data sets currently being obtained can provide unprecedented insight regarding intact cardiac anatomical structure, faithfully transferring this information into a finite element mesh that is both of good quality and is computationally tractable, is a significant challenge. A widely used approach is based on a recently published image-based unstructured mesh generation technique (Prassl et al., 2009) or its commercial implementation Tarantula (www.meshing.at, CAE-Software Solutions, Eggenburg, Austria). This method uses a modified dual mesh of an Octree applied directly to segmented 3D image stacks. The algorithm operates fully automatically with no requirements for interactivity and generates accurate volume-preserving representations of arbitrarily complex geometries with smooth surfaces. The generated unstructured meshes are hybrid, hexahedra-dominant, boundary fitted, locally refined, conformal finite element meshes (see Fig. 5, middle panel). The smooth nature of the surfaces ensures general applicability of the meshes generated, in particular for studies involving the application of strong external stimuli, since the smooth, unstructured grids lack jagged boundaries that can introduce spurious currents due to tip effects, as is the case for structured grids. To reduce the overall computational load of the meshes, unstructured grids can be generated adaptively such that the spatial resolution varies throughout the domain. Fine discretizations with little adaptivity can be used to model the myocardium thus minimizing undesired effects of grid granularity on propagation velocity, whilst coarser elements that grow in size with distance from myocardial surfaces are generated to represent a surrounding volume conductor (e.g. tissue bath or torso for example). Using adaptive mesh generation techniques facilitates the execution of bidomain simulations with a minimum of overhead due to the discretization of a surrounding volume conductor.

\subsection{Numerical schemes}

Among the possible castings of the bidomain equations, the one presented as (6) and (7) is the most popular. In the most general case, where a conducting medium is in contact with the 

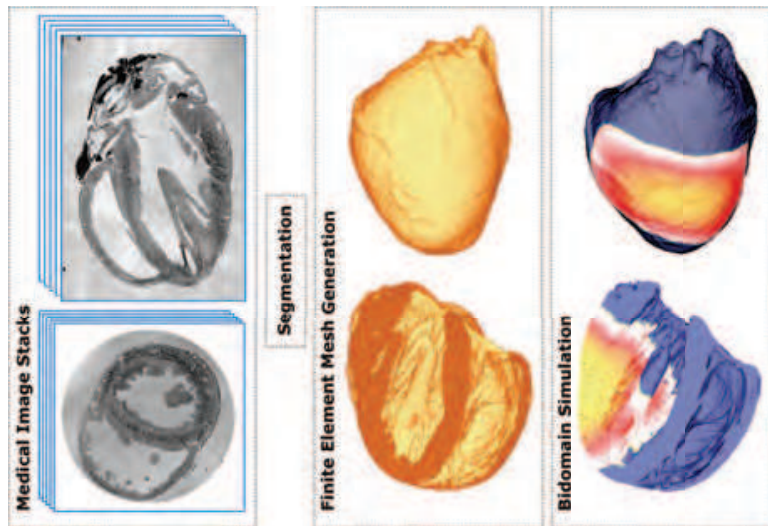

Fig. 5. Image based mesh generation pipeline for constructing geometrically detailed models of cardiac anatomy. Medical image stacks are segmented and fed into an image-based mesh generation algorithm which tessellates classified objects in the image stack into finite element meshes which are suitable for being used in bidomain simulations.

myocardium, the bidomain equations are written as

$$
\begin{aligned}
{\left[\begin{array}{c}
-\nabla \cdot\left(\boldsymbol{\sigma}_{i}+\boldsymbol{\sigma}_{\boldsymbol{e}}\right) \nabla \phi_{e} \\
-\nabla \cdot \sigma_{b} \nabla \phi_{e}
\end{array}\right]=} & {\left[\begin{array}{c}
\nabla \cdot \boldsymbol{\sigma}_{\boldsymbol{i}} \nabla V_{m} \\
I_{e}
\end{array}\right] } \\
\frac{\partial V_{m}}{\partial t}= & \frac{1}{\beta C_{m}}\left(\nabla \cdot \boldsymbol{\sigma}_{i} \nabla V_{m}+\nabla \cdot \boldsymbol{\sigma}_{\boldsymbol{i}} \nabla \phi_{e}\right) \\
& -\frac{1}{C_{m}} I_{i o n}\left(V_{m}, \boldsymbol{\eta}\right) \\
\frac{d \boldsymbol{\eta}}{d t}= & g\left(V_{m}, \boldsymbol{\eta}\right)
\end{aligned}
$$

Numerically, the bidomain equations can be solved as a coupled system (Vigmond et al., 2002) or alternatively, operator splitting techniques are applied (Keener \& Bogar, 1998) to decouple the computing scheme into three components, an elliptic partial differential equation (PDE), a parabolic PDE, and a set of non-linear ODEs. It has been shown that the decoupled scheme converges quickly against the coupled scheme by employing a Block Gauss-Seidel iteration (Pennacchio \& Simoncini, 2002). However, in most studies the components are essentially treated as independent. Solutions are then found by leap-frogging between the decoupled components where either $V_{m}$ in (9) or $\Phi_{e}$ in (10) are considered as constant. In (Vigmond et al., 2002) it has been found that with small error tolerances the differences between coupled and decoupled approaches are negligible.

Discretizing the decoupled bidomain equations leads to a three-step scheme, which involves a solution of the parabolic PDE, the elliptic PDE and the non-linear system of ODEs at each time step. The inner loop of this scheme is given by

$$
\begin{aligned}
V_{m}^{k^{*}} & =V_{m}^{k}+\frac{\Delta t}{\beta C_{m}}\left[\theta \nabla \cdot\left(\boldsymbol{\sigma}_{\boldsymbol{i}} \nabla V_{m}^{k^{*}}\right)+(1-\theta) \nabla \cdot\left(\boldsymbol{\sigma}_{\boldsymbol{i}} \nabla V_{m}^{k}\right)\right] \\
\boldsymbol{\eta}_{f}^{k+1} & =\boldsymbol{\eta}_{f}^{k} e^{-\frac{\Delta t}{\tau}}+\boldsymbol{\eta}_{\infty}\left(1-e^{-\frac{\Delta t}{\tau}}\right)
\end{aligned}
$$




$$
\begin{aligned}
\boldsymbol{\eta}_{s}^{k+1} & =\boldsymbol{\eta}_{s}^{k}+\boldsymbol{g}\left(V_{m}^{k^{*}}, \boldsymbol{\eta}_{s}^{k}\right) \Delta t \\
V_{m}^{k+1} & =V_{m}^{k^{*}}-\frac{\Delta t}{C_{m}} I_{i o n}\left(V_{m}^{k^{*}}, \boldsymbol{\eta}^{k+1}\right) \\
-\nabla \cdot\left(\left(\boldsymbol{\sigma}_{\boldsymbol{i}}+\boldsymbol{\sigma}_{\boldsymbol{e}}\right) \nabla \Phi_{e}^{k+1}\right) & =\nabla \cdot\left(\boldsymbol{\sigma}_{\boldsymbol{i}} \nabla V^{k+1}\right) \\
-\nabla \cdot\left(\sigma_{b} \nabla \Phi_{e}^{k+1}\right) & =I_{e},
\end{aligned}
$$

where the reaction and diffusion part of the parabolic PDE is split by employing a Strang or a Gudunov scheme (Qu \& Garfinkel, 1999; Sundnes et al., 2005). The parabolic portion (12) is solved either by choosing $\theta=0.5$, which results in a Crank-Nicholson scheme, or $\theta=0.0$, which results in an explicit forward Euler scheme. Depending on the choice of $\theta$ the overall system is solved then either with a fully explicit scheme, or an implicit-explicit (IMEX) scheme. The ODE integration approach in (13)-(14) is based on the Rush-Larsen method (Rush \& Larsen, 1978) where an analytical solution was used to update the fast gating variables, $\boldsymbol{\eta}_{f}$, where $\tau$ and $\boldsymbol{\eta}_{\infty}$ are functions of the rate coefficients which govern channel gating, and an explicit Euler step to update all other slower state variables, $\boldsymbol{\eta}_{s}$ (Maclachlan et al., 2007; Plank et al., 2008).

Typically, additional Dirichlet boundary conditions have to be enforced for the elliptic PDE to eliminate the Nullspace, otherwise the elliptic system is singular. This is usually achieved by adding a grounding electrode, i.e. choosing nodes in the mesh where $\phi_{e}$ is set to zero, which serves as the reference potential when simulating the shock, as it is the case in a real physical setup.

\subsection{Linear solvers}

Although the PDEs are solved most efficiently with direct methods, this is possible for small grids only (Plank et al., 2007; Vigmond et al., 2002); otherwise memory demands increase quickly which, in turn, significantly increases the required number of operations per solver step. Although direct methods have been implemented to run in parallel environments (Amestoy et al., 2001; Li \& Demmel, 2003), typically they are harder to parallelize due to the fine-grained parallelism, which is communication-intense. For large systems, iterative methods are mandatory.

When executing bidomain simulations on sequential computers, the main computational burden can be attributed to the solution of the elliptic problem and the set of ODEs. Typically, with simple ionic models, the elliptic problem contributes more than $90 \%$ to the overall workload, whereas with recent ionic models involving very stiff ODEs (Cortassa et al., 2006; Iyer et al., 2004), the ODE solution may even begin to dominate the computations. The parabolic problem is typically less of a concern. On coarser meshes, where time steps are limited by the ODEs, simple forward Euler steps can be employed to update $V_{m}$. In this case, the contributions of the diffusional component (PDE) and the local membrane component to changes in $V_{m}$ can be updated separately, which renders the PDE linear. On finer grids, semi-implicit Crank-Nicholson schemes perform well. Even when relatively cheap iterative solvers are employed, the parabolic portion can be updated efficiently due to the diagonal dominance of the linear system.

For large systems, on the order of several hundreds of thousands of unknowns, parallel computing approaches are necessary to reduce execution times. The parallel computing 
context alleviate the problem of solving the set of ODEs. State variables in an ionic model do not diffuse, which qualifies the ODEs as an embarrassingly parallel problem. No communication between processors is required to update the state variable and thus the parallel scaling of the ODE portion is linear. The parabolic problem is efficiently solved in parallel as well (Niederer S. et al., 2011). Either only a forward Euler step is required (essentially a matrix-vector product for which good scalability is expected), or the well-posed diagonally-dominant linear system is solved efficiently with relatively cheap iterative methods, such as preconditioned conjugate-gradient (CG). Typically, with an incomplete LU (ILU) preconditioner for the iterative CG solver the parabolic problem can be solved in less than 10 iterations.

The elliptic PDE is the most challenging problem. Standard iterative solvers like ILU-CG typically require several hundreds of iterations to converge, which makes this solution significantly more expensive than that of the parabolic system, although both systems share the same sparsity pattern. The parallel scaling of standard iterative solvers is fairly good (Plank et al., 2007); for instance, a parallel ILU-CG solver, where the system is decomposed by a Block Jacobi preconditioner with ILU(0), i.e. an incomplete LU factorization with zero fill-in levels that preserves the sparsity pattern of the original matrix, used as a subblock preconditioner, exhibits good parallel scaling (Plank et al., 2007). With fewer number of processors, ILU(N) with $N$ levels of fill-in tends to be more efficient, however, with an increasing number of processors the efficiency of the preconditioning deteriorates since the preconditioner is applied to the main diagonal block only. This can be circumvented by employing overlapping block preconditioners such as additive Schwarz methods, however, they increase the communication burden, which, depending on the particular hardware, may be undesirable.

It has been demonstrated in several recent studies (Austin et al., 2006; Plank et al., 2007; Weber dos Santos et al., 2004) that multilevel preconditioners for CG methods both significantly improve the overall performance and show reasonable parallel efficiency (better than $80 \%$ ) for up to 128 processors. A generally applicable algebraic multigrid preconditioner (AMG) in conjunction with an iterative Krylov solver reduces the number of iterations per solver step by almost two orders of magnitude compared to ILU-CG. Although a single iteration with AMG is significantly more expensive than with ILU, the reduction in number of iterations clearly favors a multilevel approach. In (Plank et al., 2007), a speedup of 6 was reported. Using AMG-CG is, to date, the most efficient method for solving the elliptic portion of the bidomain equations. The method is particularly well suited for defibrillation studies since it is computationally efficient and handles unstructured grids straightforwardly.

\section{References}

Adgey, A. A. J., Spence, M. S. \& Walsh, S. J. (2005). Theory and practice of defibrillation: (2) defibrillation for ventricular fibrillation., Heart 91(1): 118-125.

Aguel, F., DeBruin, K. A., Krassowska, W. \& Trayanova, N. A. (1999). Effects of electroporation on the transmembrane potential distribution in a two-dimensional bidomain model of cardiac tissue, 10: 701-714.

Amestoy, P., Duff, I. S., L'Excellent, J.-Y. \& Koster, J. (2001). Mumps: A general purpose distributed memory sparse solver, PARA '00: Proceedings of the 5th International Workshop on Applied Parallel Computing, New Paradigms for HPC in Industry and Academia, Springer-Verlag, London, UK, pp. 121-130. 
Ashihara, T., Constantino, J. \& Trayanova, N. A. (2008). Tunnel propagation of postshock activations as a hypothesis for fibrillation induction and isoelectric window., Circ Res 102(6): 737-45.

Ashihara, T. \& Trayanova, N. (2004). Asymmetry in membrane responses to electrical shocks: insight from bidomain simulations, 87: 2271-2282.

Austin, T. M., Trew, M. L. \& Pullan, A. J. (2006). Solving the cardiac bidomain equations for discontinuous conductivities., IEEE Trans Biomed Eng 53(7): 1265-72.

Bardy, G. H., Hofer, B., Johnson, G., Kudenchuk, P. J., Poole, J. E., Dolack, G. L., Gleva, M., Mitchell, R. \& Kelso, D. (1993). Implantable transvenous cardioverter-defibrillators., Circulation 87(4): 1152-68.

Bishop, M., Rodriguez, B., Qu, F., Efimov, I., Gavaghan, D. \& Trayanova, N. (2007). The role of photon scattering in optical signal distortion during arrhythmia and defibrillation, Biophys. J. 93: 3714-3726.

Buist, M., Sands, G., Hunter, P. \& Pullan, A. (2003). A deformable finite element derived finite difference method for cardiac activation problems., Ann Biomed Eng 31(5): 577-88.

Burton, R., Plank, G., Schneider, J., Grau, V., Ahammer, H., Keeling, S., Lee, J., Smith, N., Gavaghan, D., Trayanova, N. \& Kohl, P. (2006). 3-d models of individual cardiac histo-anatomy: tools and challenges, Ann NY Acad Sci 1380: 301-319.

Cheng, D. K.-L., Tung, L. \& Sobie, E. A. (1999). Nonuniform responses of transmembrane potential during electric field stimulation of single cardiac cells, 277: H351-H362.

Cheng, Y., Mowrey, K. A., Van Wagoner, D. R., Tchou, P. J. \& Efimov, I. R. (1999). Virtual electrode induced re-excitation: a mechanism of defibrillation, 85: 1056-1066.

Clayton, R. H., Bernus, O., Cherry, E. M., Dierckx, H., Fenton, F. H., Mirabella, L., Panfilov, A. V., Sachse, F. B., Seemann, G. \& Zhang, H. (2011). Models of cardiac tissue electrophysiology: progress, challenges and open questions., Prog Biophys Mol Biol 104(1-3): 22-48.

Clerc, L. (1976). Directional differences of impulse spread in trabecular muscle from mammalian heart, Journal of Physiology 255: 335-346.

Cortassa, S., Aon, M. A., O’Rourke, B., Jacques, R., Tseng, H. J., Marban, E. \& Winslow, R. L. (2006). A computational model integrating electrophysiology, contraction, and mitochondrial bioenergetics in the ventricular myocyte., Biophys J 91(4): 1564-89.

Courant, R., Friedrichs, K. \& Lewy, H. (1928). Über die partiellen Differenzengleichungen der mathematischen Physik, Mathematische Annalen 100(1): 32-74.

DeBruin, K. A. \& Krassowska, W. (1998). Electroporation and shock-induced transmembrane potential in a cardiac fiber during defibrillation strength shocks, 26: 584-596.

Desplantez, T., Dupont, E., Severs, N. J. \& Weingart, R. (2007). Gap junction channels and cardiac impulse propagation., J Membr Biol 218(1-3): 13-28.

Efimov, I. R., Aguel, F., Cheng, Y., Wollenzier, B. \& Trayanova, N. A. (2000). Virtual electrode polarization in the far field: implications for external defibrillation, 279: H1055-H1070.

Efimov, I. R., Cheng, Y., Biermann, M., Van Wagoner, D. R., Mazgalev, T. N. \& Tchou, P. J. (1997). Transmembrane voltage changes produced by real and virtual electrodes during monophasic defibrillation shock delivered by an implantable electrode, 8: 1031-45.

Efimov, I. R., Gray, R. A. \& Roth, B. J. (2000). Virtual electrodes and deexcitation: new insights into fibrillation induction and defibrillation, 11: 339-353. 
Entcheva, E., Trayanova, N. A. \& Claydon, F. J. (1999). Patterns of and mechanisms for shock-induced polarization in the heart: a bidomain analysis., IEEE Trans Biomed Eng 46(3): 260-70.

Evans, F. G., Ideker, R. E. \& Gray, R. A. (2002). Effect of shock-induced changes in transmembrane potential on reentrant waves and outcome during cardioversion of isolated rabbit hearts, 13: 1118-1127.

Fenton, F. H., Cherry, E. M., Karma, A. \& Rappel, W. J. (2005). Modeling wave propagation in realistic heart geometries using the phase-field method., Chaos 15(1): 13502.

Franzone, P. C., Deuflhard, P., Erdmann, B., Lang, J. \& Pavarino, L. F. (2006). Adaptivity in space and time for reaction-diffusion systems in electrocardiology, SIAM J. Sci. Comput. 28(3): 942-962.

Frazier, D. W., Wolf, P. D., Wharton, J. M., Tang, A. S., Smith, W. M. \& Ideker, R. E. (1989). Stimulus-induced critical point. mechanism for electrical initiation of reentry in normal canine myocardium, J Clin Invest 83: 1039-52.

Gourdie, R. G., Green, C. R. \& Severs, N. J. (1991). Gap junction distribution in adult mammalian myocardium revealed by an anti-peptide antibody and laser scanning confocal microscopy., J Cell Sci 99 ( Pt 1): 41-55.

Hand, P. E., Griffith, B. E. \& Peskin, C. S. (2009). Deriving Macroscopic Myocardial Conductivities by Homogenization of Microscopic Models., Bull Math Biol .

Harrild, D. \& Henriquez, C. (2000). A computer model of normal conduction in the human atria., Circ Res 87(7): E25-36.

Harrild, D. M. \& Henriquez, C. S. (1997). A finite volume model of cardiac propagation., Ann Biomed Eng 25(2): 315-34.

Henriquez, C. S. (1993). Simulating the elctrical behavior of cardiac tissue using the bidomain equations, Critical Reviews in Biomedical Engineering 1(1): 1-77.

Hooke, N., Henriquez, C. S., Lanzkron, P. \& Rose, D. (1994). Linear algebraic transformations of the bidomain equations: implications for numerical methods., Math Biosci 120(2): 127-45.

Hooks, D. A., Trew, M. L., Caldwell, B. J., Sands, G. B., LeGrice, I. J. \& Smaill, B. H. (2007). Laminar arrangement of ventricular myocytes influences electrical behavior of the heart., Circ Res 101(10): e103-12.

Hoyt, R. H., Cohen, M. L. \& Saffitz, J. E. (1989). Distribution and three-dimensional structure of intercellular junctions in canine myocardium., Circ Res 64(3): 563-74.

Ideker, R. E., Wolf, P. D., Alferness, C., Krassowska, W. \& Smith, W. M. (1991). Current concepts for selecting the location, size and shape of defibrillation electrodes, Pacing Clin Electrophysiol 14: 227-40.

Iyer, V., Mazhari, R. \& Winslow, R. L. (2004). A computational model of the human left-ventricular epicardial myocyte., Biophys J 87(3): 1507-25.

Keener, J. P. \& Bogar, K. (1998). A numerical method for the solution of the bidomain equations in cardiac tissue., Chaos 8(1): 234-241.

Kleber, A. G. \& Rudy, Y. (2004). Basic mechanisms of cardiac impulse propagation and associated arrhythmias., Physiol Rev 84(2): 431-88.

Knisley, S. B., Trayanova, N. A. \& Aguel, F. (1999). Roles of electric field and fiber structure in cardiac electric stimulation, 77: 1404-1417.

Knisley, S., Smith, W. \& Ideker, R. (1994). Prolongation and shortening of action potentials by electrical shocks in frog ventricular muscle., Am J Physiol 266 (6 Pt 2): H2348-58. 
LeGrice, I., Smaill, B., Chai, L., Edgar, S., Gavin, J. \& Hunter, P. (1995). Laminar structure of the heart: ventricular myocyte arrangement and connective tissue architecture in the dog., Am. J. Physiol. 269: H571-H582.

Leon, L. J. \& Roberge, F. A. (1991). Directional characteristics of action potential propagation in cardiac muscle. A model study., Circ Res 69(2): 378-95.

Li, X. \& Demmel, J. (2003). SuperLU DIST: A scalable distributed-memory sparse direct solver for unsymmetric linear systems, ACM Transact ions on Mathematical Software (TOMS) 29(2): 110-140.

Maclachlan, M. C., Sundnes, J. \& Spiteri, R. J. (2007). A comparison of non-standard solvers for ODEs describing cellular reactions in the heart., Comput Methods Biomech Biomed Engin 10(5): 317-26.

Maisel, W. (2006). Pacemaker and ICD generator reliability: meta-analysis of device registries., JAMA 295: 1929-1934.

Munteanu, M., Pavarino, L. \& Scacchi, S. (2009). A scalable Newton-Krylov-Schwarz method for the bidomain reaction-diffusion system, SIAM J. Sci. Comput. .

Neu, J. \& Krassowska, W. (1993). Homogenization of syncytial tissue, Critical Reviews in Biomedical Engineering 21(2): 137-199.

Niederer S., Mitchell L., Smith N. \& Plank G. (2011). Simulating human cardiac electrophysiology on clinical time scales, Front Physiol 2:14.

Pennacchio M., Savare G. \& Colli Franyone P. (2006). Multiscale modeling for the bioelectric activity of the heart., SIAM J. Math. Anal 37(4): 1333-1370.

Pennacchio, M. \& Simoncini, V. (2002). Efficient algebraic solution of reaction-diffusion systems for the cardiac excitation process, J Comp Appl Math 145: 49-70.

Plank, G., Burton, R., Hales, P., Bishop, M., Mansoori, T., Bernabeau, M., Garny, A., Prassl, A., Bollensdorf, C., Mason, F., Rodriguez, B., Grau, V., Schneider, J., Gavaghan, D. \& Kohl, P. (2009). Generation of histo-anatomically representative models of the individual heart: tools and application, Phil Trans Roc Soc.

Plank, G., Leon, L.J., Kimber, S. \& Vigmond, E. J. (2005). Defibrillation depends on Conductivity Fluctuations and the Degree of Disorganization in Reentry Patterns, J Cardiovasc Electrophysiol 16(2):205-16.

Plank, G., Liebmann, M., Weber dos Santos, R., Vigmond, E. J. \& Haase, G. (2007). Algebraic Multigrid Preconditioner for the Cardiac Bidomain Model, IEEE TBME 54(4):585-96.

Plank, G., Zhou, L., Greenstein, J. L., Cortassa, S., Winslow, R. L., O'Rourke, B. \& Trayanova, N. A. (2008). From mitochondrial ion channels to arrhythmias in the heart: computational techniques to bridge the spatio-temporal scales., Philos Transact $A$ Math Phys Eng Sci 366(1879): 3381-409.

Plonsey, R. (1988). Bioelectric sources arising in excitable fibers (ALZA lecture)., Ann Biomed Eng 16(6): 519-46.

Pollard, A. E., Burgess, M. J. \& Spitzer, K. W. (1993). Computer simulations of three-dimensional propagation in ventricular myocardium. Effects of intramural fiber rotation and inhomogeneous conductivity on epicardial activation., Circ Res 72(4): 744-56.

Pollard, A. E., Hooke, N. \& Henriquez, C. S. (1992). Cardiac propagation simulation., Crit Rev Biomed Eng 20(3-4): 171-210.

Potse, M., Dube, B., Richer, J., Vinet, A. \& Gulrajani, R. M. (2006). A comparison of monodomain and bidomain reaction-diffusion models for action potential propagation in the human heart., IEEE Trans Biomed Eng 53(12 Pt 1): 2425-35. 
Prassl, A. J., Kickinger, F., Ahammer, H., Grau, V., Schneider, J. E., Hofer, E., Vigmond, E. J., Trayanova, N. A. \& Plank, G. (2009). Automatically generated, anatomically accurate meshes for cardiac electrophysiology problems., IEEE Trans Biomed Eng 56(5): 1318-30.

Qu, Z. \& Garfinkel, A. (1999). An advanced algorithm for solving partial differential equation in cardiac conduction., IEEE Trans Biomed Eng 46(9): 1166-8.

Roberts, D. E. \& Scher, A. M. (1982). Effect of tissue anisotropy on extracellular potential fields in canine myocardium in situ., Circ Res 50(3): 342-51.

Roberts, S. F., Stinstra, J. G. \& Henriquez, C. S. (2008). Effect of nonuniform interstitial space properties on impulse propagation: a discrete multidomain model., Biophys J 95(8): 3724-37.

Rocha, B. M., Kickinger, F., Prassl, A. J., Haase, G., Vigmond, E. J., Santos, R. W., Zaglmayr, S. \& Plank, G. (2011). A macro finite-element formulation for cardiac electrophysiology simulations using hybrid unstructured grids., IEEE Trans Biomed Eng 58(4): 1055-65.

Rodriguez, B., Li, L., Eason, J. C., Efimov, I. R. \& Trayanova, N. A. (2005). Differences between left and right ventricular chamber geometry affect cardiac vulnerability to electric shocks., Circ Res 97(2): 168-75.

Rogers, J. M. \& McCulloch, A. D. (1994). A collocation-Galerkin finite element model of cardiac action potential propagation., IEEE Trans Biomed Eng 41(8): 743-57.

Roth, B. J. (1995). A mathematical model of make and break electrical stimulation of cardiac tissue by a unipolar anode or cathode, IEEE Transactions on Biomedical Engineering 42: 1174-1184.

Roth, B. J. (1997). Electrical conductivity values used with the bidomain model of cardiac tissue., IEEE Trans Biomed Eng 44(4): 326-8.

Roth, B. J. \& Krassowska, W. (1998). The induction of reentry in cardiac tissue. The missing link: How electric fields alter transmembrane potential., Chaos 8(1): 204-220.

Rush, S. \& Larsen, H. (1978). A practical algorithm for solving dynamic membrane equations., IEEE Trans Biomed Eng 25(4): 389-92.

Saucerman, J. J., Healy, S. N., Belik, M. E., Puglisi, J. L. \& McCulloch, A. D. (2004). Proarrhythmic consequences of a KCNQ1 AKAP-binding domain mutation: computational models of whole cells and heterogeneous tissue., Circ Res 95(12): 1216-24.

Seemann, G., Hoper, C., Sachse, F. B., Dossel, O., Holden, A. V. \& Zhang, H. (2006). Heterogeneous three-dimensional anatomical and electrophysiological model of human atria., Philos Transact A Math Phys Eng Sci 364(1843): 1465-81.

Sepulveda, N. G., Roth, B. J. \& Wikswo, Jr, J. P. (1989). Current injection into a two-dimensional anisotropic bidomain., Biophys J 55(5): 987-99.

Severs, N. J., Bruce, A. F., Dupont, E. \& Rothery, S. (2008). Remodelling of gap junctions and connexin expression in diseased myocardium., Cardiovasc Res 80(1): 9-19.

Skouibine, K., Trayanova, N. A. \& Moore, P. (2000a). Success and failure of the defibrillation shock: insights from a simulation study, 11: 785-796.

Skouibine, K., Trayanova, N. A. \& Moore, P. K. (2000b). A numerically efficient model for simulation of defibrillation in an active bidomain sheet of myocardium, 166: 85-100.

Sobie, E. A., Susil, R. C. \& Tung, L. (1997). A generalized activating function for predicting virtual electrodes in cardiac tissue., Biophys J 73(3): 1410-23. 
Spach, M. S. \& Heidlage, J. F. (1995). The stochastic nature of cardiac propagation at a microscopic level. Electrical description of myocardial architecture and its application to conduction., Circ Res 76(3): 366-80.

Streeter, D., Spontnitz, H., Patel, D., Ross, J. \& Sonnenblick, E. (1969). Fiber orientation in the canine left ventricle during diastole and systole, Circ. Res. 24: 339-347.

Sundnes, J., Lines, G. T. \& Tveito, A. (2005). An operator splitting method for solving the bidomain equations coupled to a volume conductor model for the torso., Math Biosci 194(2): 233-48.

Sundnes, J., Nielsen, B. F., Mardal, K. A., Cai, X., Lines, G. T. \& Tveito, A. (2006). On the Computational Complexity of the Bidomain and Monodomain Models of Electrophysiology, Ann. of Biomed. Eng. 34(7): 1088-1097.

Ten Tusscher, K. H., Hren, R. \& Panfilov, A. V. (2007). Organization of ventricular fibrillation in the human heart., Circ Res 100(12): e87-101.

Trayanova, N., Skouibine, K. \& Aguel, F. (1998). The role of cardiac tissue structure in defibrillation., Chaos 8(1): 221-233.

Trew, M. L., Smaill, B. H., Bullivant, D. P., Hunter, P. J. \& Pullan, A. J. (2005). A generalized finite difference method for modeling cardiac electrical activation on arbitrary, irregular computational meshes., Math Biosci 198(2): 169-89.

Trew, M., Le Grice, I., Smaill, B. \& Pullan, A. (2005). A finite volume method for modeling discontinuous electrical activation in cardiac tissue., Ann Biomed Eng 33(5): 590-602.

Vigmond, E. J., Aguel, F. \& Trayanova, N. A. (2002). Computational techniques for solving the bidomain equations in three dimensions., IEEE Trans Biomed Eng 49(11): 1260-9.

Vigmond, E. J., Tsoi, V., Kuo, S., Arevalo, H., Kneller, J., Nattel, S. \& Trayanova, N. (2004). The effect of vagally induced dispersion of action potential duration on atrial arrhythmogenesis., Heart Rhythm 1(3): 334-44.

Virag, N., Jacquemet, V., Henriquez, C. S., Zozor, S., Blanc, O., Vesin, J. M., Pruvot, E. \& Kappenberger, L. (2002). Study of atrial arrhythmias in a computer model based on magnetic resonance images of human atria., Chaos 12(3): 754-763.

Wang, S., Leon, L. J. \& Roberge, F. A. (1996). Interactions between adjacent fibers in a cardiac muscle bundle., Ann Biomed Eng 24(6): 662-74.

Weber dos Santos, R., Plank, G., Bauer, S. \& Vigmond, E. (2004). Parallel multigrid preconditioner for the cardiac bidomain model., IEEE Trans Biomed Eng 51(11): 1960-8.

Wikswo Jr, J. P., Lin, S.-F. \& Abbas, R. A. (1995). Virtual electrodes in cardiac tissue: a common mechanism for anodal and cathodal stimulation, 69: 2195-2210.

Zhou, X., Daubert, J. P., Wolf, P. D., Smith, W. M. \& Ideker, R. E. (1993). Epicardial mapping of ventricular defibrillation with monophasic and biphasic shocks in dogs., Circ Res 72(1): 145-160.

Zipes, D. P., Wyse, D. G., Friedman, P. L., Epstein, A. E., Hallstrom, A. P., Greene, H. L., Schron, E. B. \& Domanski, M. (1997). A comparison of antiarrhythmic-drug therapy with implantable defibrillators in patients resuscitated from near-fatal ventricular arrhythmias. The Antiarrhythmics versus Implantable Defibrillators (AVID) Investigators., N Engl J Med 337(22): 1576-83. 


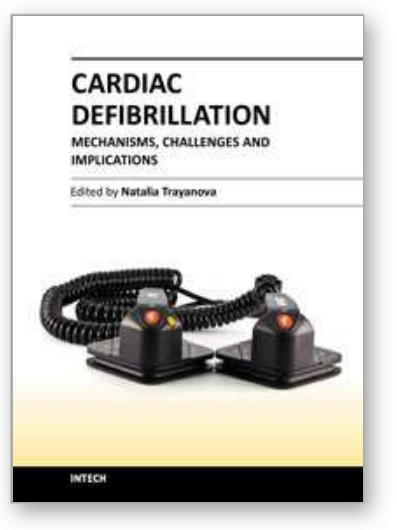

\author{
Cardiac Defibrillation - Mechanisms, Challenges and Implications \\ Edited by Prof. Natalia Trayanova
}

ISBN 978-953-307-666-9

Hard cover, 248 pages

Publisher InTech

Published online 26, September, 2011

Published in print edition September, 2011

The only known effective therapy for lethal disturbances in cardiac rhythm is deï-brillation, the delivery of a strong electric shock to the heart. This technique constitutes the most important means for prevention of sudden cardiac death. The efficacy of defibrillation has led to an exponential growth in the number of patients receiving implantable devices. The objective of this book is to present contemporary views on the basic mechanisms by which the heart responds to an electric shock, as well as on the challenges and implications of clinical defibrillation. Basic science chapters elucidate questions such as lead configurations and the reasons by which a defibrillation shock fails. Chapters devoted to the challenges in the clinical procedure of defibrillation address issues related to inappropriate and unnecessary shocks, complications associated with the implantation of cardioverter/defibrillator devices, and the application of the therapy in pediatric patients and young adults. The book also examines the implications of defibrillation therapy, such as patient risk stratification, cardiac rehabilitation, and remote monitoring of patient with implantable devices.

\title{
How to reference
}

In order to correctly reference this scholarly work, feel free to copy and paste the following:

Gernot Plank and Natalia Trayanova (2011). Modeling Defibrillation, Cardiac Defibrillation - Mechanisms, Challenges and Implications, Prof. Natalia Trayanova (Ed.), ISBN: 978-953-307-666-9, InTech, Available from: http://www.intechopen.com/books/cardiac-defibrillation-mechanisms-challenges-and-implications/modelingdefibrillation

\section{INTECH}

open science | open minds

\section{InTech Europe}

University Campus STeP Ri

Slavka Krautzeka 83/A

51000 Rijeka, Croatia

Phone: +385 (51) 770447

Fax: +385 (51) 686166

www.intechopen.com

\section{InTech China}

Unit 405, Office Block, Hotel Equatorial Shanghai

No.65, Yan An Road (West), Shanghai, 200040, China

中国上海市延安西路65号上海国际贵都大饭店办公楼405单元

Phone: +86-21-62489820

Fax: $+86-21-62489821$ 
(C) 2011 The Author(s). Licensee IntechOpen. This chapter is distributed under the terms of the Creative Commons Attribution-NonCommercialShareAlike-3.0 License, which permits use, distribution and reproduction for non-commercial purposes, provided the original is properly cited and derivative works building on this content are distributed under the same license. 